\title{
Leaf anatomy of Cyperus species growing in different soils of Punjab Pakistan
}

\author{
Aamir Mumtaz $^{*}$, Aisha Taseen ${ }^{1}$ and Amina Ameer ${ }^{1}$ \\ 1. Government Post Graduate College Mianwali-Pakistan \\ *Corresponding author's email: aamirkazmi038@gmail.com \\ Citation \\ AamirMumtaz, Aisha Taseen and Amina Ameer. Leaf anatomy of Cyperus species growing in different soils of \\ Punjab Pakistan. Pure and Applied Biology. Vol. 10, Issue 3, pp726-737. \\ http://dx.doi.org/10.19045/bspab.2021.100074
}

Received: 21/09/2020 Revised: 20/11/2020

Accepted: 00/11/2020

Online First: 01/12/2020

\section{Abstract}

Cyperaceae also called the Sedge-family is the third-largest monocot family. They have a cosmopolitan distribution with a wide variety of habitats such as steep slopes, high altitude, extreme aridity, extreme salinity, swamps, marshes, and moist areas. In this study, the transverse sections of leaves of different species of the genus Cyperus were collected from different regions of Punjab including Faisalabad, Chiniot, Jarhanwala, Sahianwala, KalarKahar, Khabeki Lake, Pakkana, and Balloki. The soil samples and leaf sections were analyzed for the adaptations in leaf anatomy in different soils of Punjab. This analysis demonstrated diverse modifications in the leaf area; thickness of vascular bundles; altered phloem and metaxylem cell area; sclerenchyma, collenchyma, and aerenchyma thickness; and variations in upper and lower epidermal cell area and the number of stomata on both sides of the epidermis, etc. based on the soil structure and composition in different regions of Punjab. The modifications were proportional to the accelerated survival and growth rate of the species in that region. Sedges are present in different environment such as degraded soil, hyper-saline waters, dry land salinity; however, many are related to Lakes or soils. The anatomical structures of various plant parts are seen as an adaptation to the ecological habitat of certain plants. It was therefore believed that indigenous species of the Cyperaceous family may have evolved specific leaf anatomy which has permitted them to live under severe environmental conditions such as drought and salinity.

Keywords: Cyperaceae; Cyperus; Leaf; Punjab; Pakistan; Soil

\section{Introduction}

Among the top ten families of angiosperms, Cyperaceae is the third-largest monocot family comprising of 4000-5000 species worldwide. All these species are categorized into more than 70 genera after a detailed analysis of features such as dorsoventrally and laterally flattened pleomorphic trimerous gynoecium, and spirally arranged legumes, etc., through ontogenetically and anatomical techniques of analysis [1]. Among the vascular plants, this is the second largest family with the number of genera above 70 . Also, it is the second-largest family of order poales. Moreover, it also occupies the second position among the families of $\mathrm{C} 4$ plants with 1500 species that make up $20 \%$ of the total C4 plants. But owing to the varying nature of $\mathrm{C} 4$ plants, these $\mathrm{C} 4$ species of Cyperaceae are also diverse in their anatomical and biochemical characteristics [2]. 
Cyperaceae is also called the sedge-family of angiosperms because of the genus Cyperus. L.containing almost 600 species that can grow in all types of temperate and tropical zones. These are perennial/annual aquatic plants that can also grow up to $0.5 \mathrm{~cm}$ in water [3]. Because of differences in the development of anatomical features, the genus can be subdivided into two sub-genera i.e., C4 type photosynthetic Cyperus with chlorocyperoid anatomy of kranz cells and C3 type Photosynthetic Cyperus with eucyperoid anatomy in the absence of kranz cells [4].

Cyperaceae family has a cosmopolitan distribution. In Pakistan, the species are distributed largely throughout from the plains of Sindh to Punjab, sandy mountains of Baluchistan, and green valleys of Kashmir, Gilgit Baltistan and Khyber Pakhtunkhwa [5]. It has been reported that over 22 genera and more than 179 species are growing throughout the country [6]. Punjab province lies $27^{\circ} 42^{\prime}$ to $34^{\circ} 02^{\prime}$ 'North latitude and $69^{\circ} 18^{\prime}$ to $75^{\circ} 23^{\prime}$ east longitudes on the globe and is bordered on West by Khyber Pakhtunkhwa, on South by Sindh, and on North by Baluchistan. It occupies 20.63 million hectares of the tropical zone and spreads $616 \mathrm{~km}$ from East to West and $1078 \mathrm{~km}$ from North to South (Table 1).

Punjab is divided into many districts and regions which have different texture and composition of the soil [7]. For example, Chakwal district has a soil structure comprising mostly of sandstone, limestone and shale rocks. So, it is weak and calcareous and also has gravels, stones, and rocks, etc. The consistency of soil in this district ranges from sandy loam to loam with a $\mathrm{pH}$ of 7.7-7.8. Similarly, the Sahianwala of Punjab has three different soil types i.e., wetland, dry saline and highly saline soil. Murree has two different soil structures comprising of reddish/purple sandstones and greyish sandstones divided into upper and lower regions. Likewise, the soil of Chiniot is sandy and that of KalarKahar is saline while Faisalabad has saline-sodic soil [8].

Studies have identified that the leaf anatomy of most of the members of the Cyperaceae family shows midrib with solid bulliform cells, abaxial epidermal cells shorter than the adaxial epidermal cells, and double-layered bundle sheath and collateral vascular bundle with outer parenchymatous layer and fibrous sclerified inner layer. Cyperaceae family also shows phytolith (silica bodies) in the epidermis. Some of the species also have Paracytic stomata with two subsidiary cells adjacent to the aperture [9].

The composition and structure of soil affect the growth of various parts of plants. Plants modify their anatomical features to sustain the challenging environments for survival and efficient growth. We designed this study to identify the anatomical changes in the leaf of Cyperaceae species growing in different soil regions of Punjab, Pakistan. The study aimed to determine the impact of soil structure on the leaf anatomy of selected species of Cyperaceae family in the selected areas of Punjab. After a detailed analysis of anatomical structures, our study found that the species diversity of Cyperus in different regions of Punjab was based on the changes in leaf anatomy of the plants due to differences in the soil texture and composition.

\section{Materials and Methods}

For this study, detailed surveys were carried out throughout the Punjab region including major cities such as Faisalabad, Rawalpindi, Murree, Sargodha, Sahianwala, Khushab, and Lahore, Mianwali, Jhang, Nowshera and some smaller districts. During these surveys, soil samples from different ecological zones pertaining to the studied areas were obtained and were subjected to laboratory analysis. Soil samples underwent chemical analysis; in addition, electrical conductivity studies for various ions such as $\mathrm{Na}+, \mathrm{K}+, \mathrm{Ca} 2+, \mathrm{Cl}$ - were also performed. 
Soil samples acquired from the Sahianwala region were categorized as three different types: 1) Dry saline soil 2) Highly saline soil 3) Wetland. Conductivity meters were employed for the analysis of electrical conductivity. The anatomical parameters were assessed using a compound microscope, a calibrated stage micrometer together with an ocular micrometer.

Formalin Acetic Acid (FAA) solution comprising of $50 \%$ ethyl alcohol, $35 \%$ distilled water, $10 \%$ acetic acid, $5 \%$ formalin, was used for material fixation. Long term fixation of tissues was done using Acetic Alcohol solution (25\% acetic acid with $75 \%$ ethyl alcohol).

Slides of the transverse section were prepared using a double-stained standard technique. The formula mentioned below was used to calculate the area of different cells and tissues (this formula is a modification of area of circle i.e. $\pi \mathrm{r} 2$ ).

Area $=\underline{\text { Maximum length } \times \text { Maximum width }} \times 22$ 28

Table 1. Habitats descriptions of collecting sites

\begin{tabular}{|c|c|c|c|}
\hline LOCATIONS & Latitude & Longitude & Altitude(Feet) \\
\hline Sahiwanla & $31^{0} 38^{\prime} 20.93^{\prime \prime}$ & $73^{0} 14^{\prime} 04.10^{\prime \prime}$ & 633 \\
\hline Faisalabad & $31^{0} 27^{\prime} 17.47^{\prime \prime}$ & $73^{0} 45^{\prime} 06.66^{\prime \prime}$ & 619 \\
\hline Sargodha & $32^{\circ} 07^{\prime} 10.33^{\prime \prime}$ & $73^{0} 33^{\prime} 42.63^{\prime \prime}$ & 592 \\
\hline Murree & $33^{\circ} 55^{\prime} 21.53^{\prime \prime}$ & $73^{0} 23^{\prime} 30.28^{\prime \prime}$ & 5909 \\
\hline Rawalpindi & $33^{0} 34^{\prime} 24.30^{\prime \prime}$ & $73^{0} 05^{\prime} 45.46^{\prime \prime}$ & 1669 \\
\hline Lahore & $31^{0} 31^{\prime} 57.27^{\prime \prime}$ & $74^{0} 2^{\prime} 45.31^{\prime \prime}$ & 712 \\
\hline Mianwali & $32^{0} 33^{\prime} 35.75^{\prime \prime}$ & $71^{0} 33^{\prime} 08.19^{\prime \prime}$ & 698 \\
\hline Khushab & $33^{0} 53^{\prime} 34.04^{\prime \prime}$ & $73^{0} 20^{\prime} 20.84^{\prime \prime}$ & 213 \\
\hline Nowshera & $32^{0} 33^{\prime} 44.31^{\prime \prime}$ & $72^{0} 09^{\prime} 33.00^{\prime \prime}$ & 2852 \\
\hline Jhang & $31^{0} 16^{\prime} 41.05^{\prime \prime}$ & $72^{0} 19^{\prime} 54.03^{\prime \prime}$ & 519 \\
\hline
\end{tabular}

\section{Statistical analysis}

Analysis of variance (ANOVA) via complete randomized design (CRD) with the twofactor factorial arrangement is used for the analysis of the data.

\section{Results}

Analysis of modifications in leaf anatomy with respect to different texture and composition of soil in various regions of Punjab showed that Cyperus laevigatus growing in the soils of Sahianwala in the dry and saline regions had increased stomatal cell area in the upper and lower epidermis of the leaf (Fig. 1g, 1h) while the leaves also had thickest sclerenchyma layer and thinnest aerenchyma as compared to other Cyperus species in the surrounding areas (Fig. 11, 1n).
Another species Cyperus glaber in the Head Rasool Region also showed modifications in leaf anatomy in the form of the thick epidermis (Fig. 1c), largest cortical cell area (Fig. 1f) and maximum phloem cell area (Fig. 1q) while the Cyperus squarossus species in the same region had maximum aerenchyma cell area when compared to other species of the same genera in Punjab (Fig. 1n).

The soils of Khabeki Lake showed anatomical changes in the leaf of Cyperus maritimus in the form of increased leaf area (Fig. 1a), thickest epidermis (Fig 1c), minimum upper epidermal cell area, the highest number of stomata on the upper epidermis (Fig. 1j), maximum aerenchyma cell area (Fig. 1n)and 
minimum stomatal cell area on lower epidermis (Fig. 1g).

Likewise, the leaf anatomy of Cyperus compressus growing in the soils of KalarKahar showed thinnest epidermis (Fig. 1c), and minimum bulliform cell area (Fig. 1k) as modifications in response to soil composition and structure.

The analysis of leaf anatomy of $C$. haspans growing in Chiniot demonstrated minimum phloem cell area (Fig. 1q), and largest bulliform cell area (Fig. 1k). Another species Cyperus difformis collected from the soils of Jarhanwala in Punjab had maximum upper epidermal cell area (Fig. 1e), maximum vascular bundle cell area (Fig. 1o) and maximum metaxylem cell areas (Fig. 1p). $C$. alternifolia collected from botanical garden of university of agriculture, Faisalabad, showed minimum leaf area (Fig. 1a), and minimum sclerenchyma thickness (Fig. 11).Another species C. rotundus collected from the same university showed large vascular bundle cell area (Fig. 1o), thin collenchyma (Fig. 1m), minimum cortical cell area (Fig. 1f), minimum

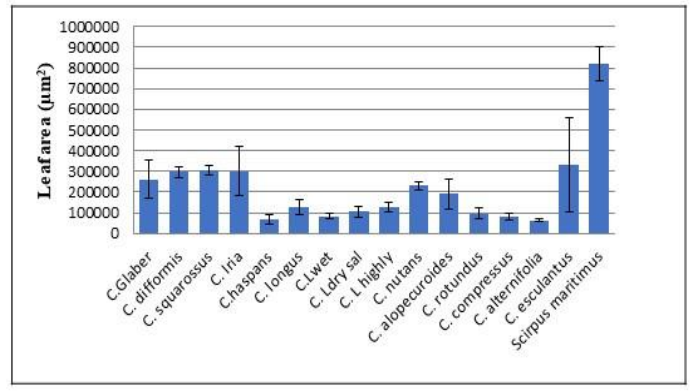

a

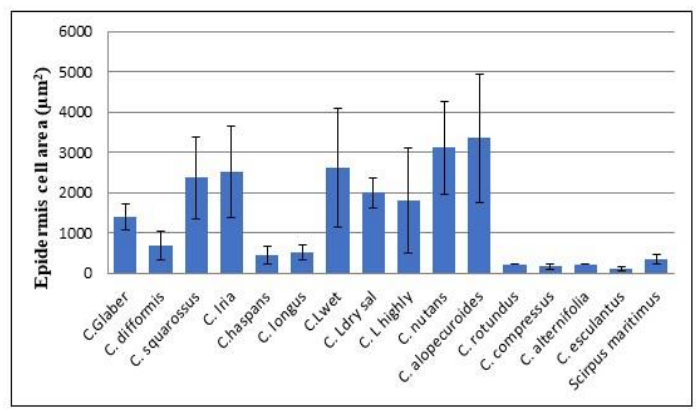

C upper epidermal stomatal cell area (Fig. 1h), and maximum number of stomata on lower epidermis (Fig. 1i).

C. nutans growing in Khari Mural also showed modifications in the leaf anatomy in the form of large lower epidermal cell area (Fig. 1d), thickest collenchyma(Fig 1m), thick bulliform cell area(Fig. 1k), minimum number of stomata on lower and upper epidermis(Fig 1i, 1j), minimum vascular bundle cell area(Fig. 10), and minimum metaxylem cell area(Fig. 1p).Cyperus esculuntus samples collected from Pakkana region showed overall minimum epidermal cell area (Fig 1b), and minimum lower epidermal cell area (Fig. 1d). Finally, a single species C.alopecuroides Collected from Balloki demonstrated maximum epidermal cell area (Fig. 1b) in leaf anatomy modifications with respect to soil in different regions of Punjab. The transverse sections of some species of Cyperaceae in Punjab have been shown in (Fig. 2).

The (Table 2) below shows statistical results, analysis of leaf anatomy modifications in various species of Cyperus in Punjab.

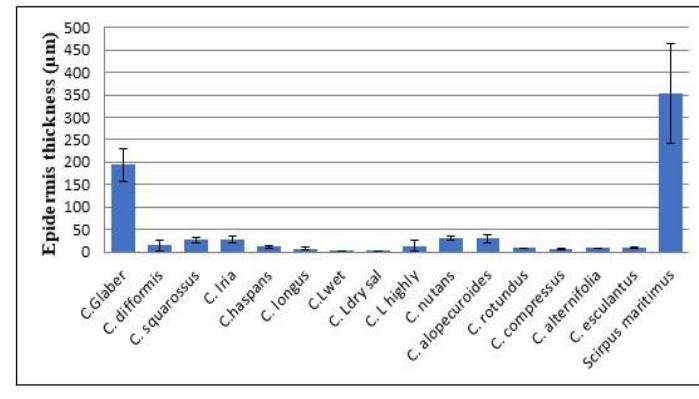

b

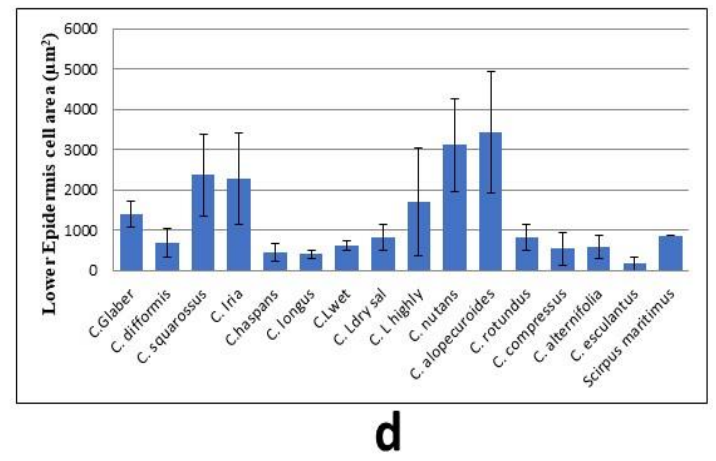




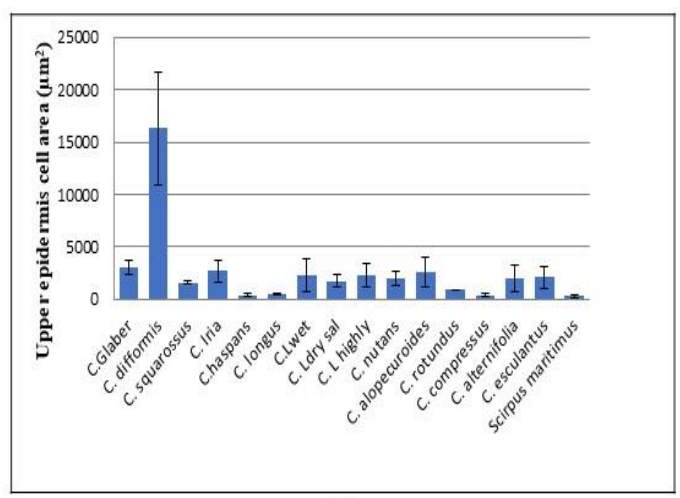

e
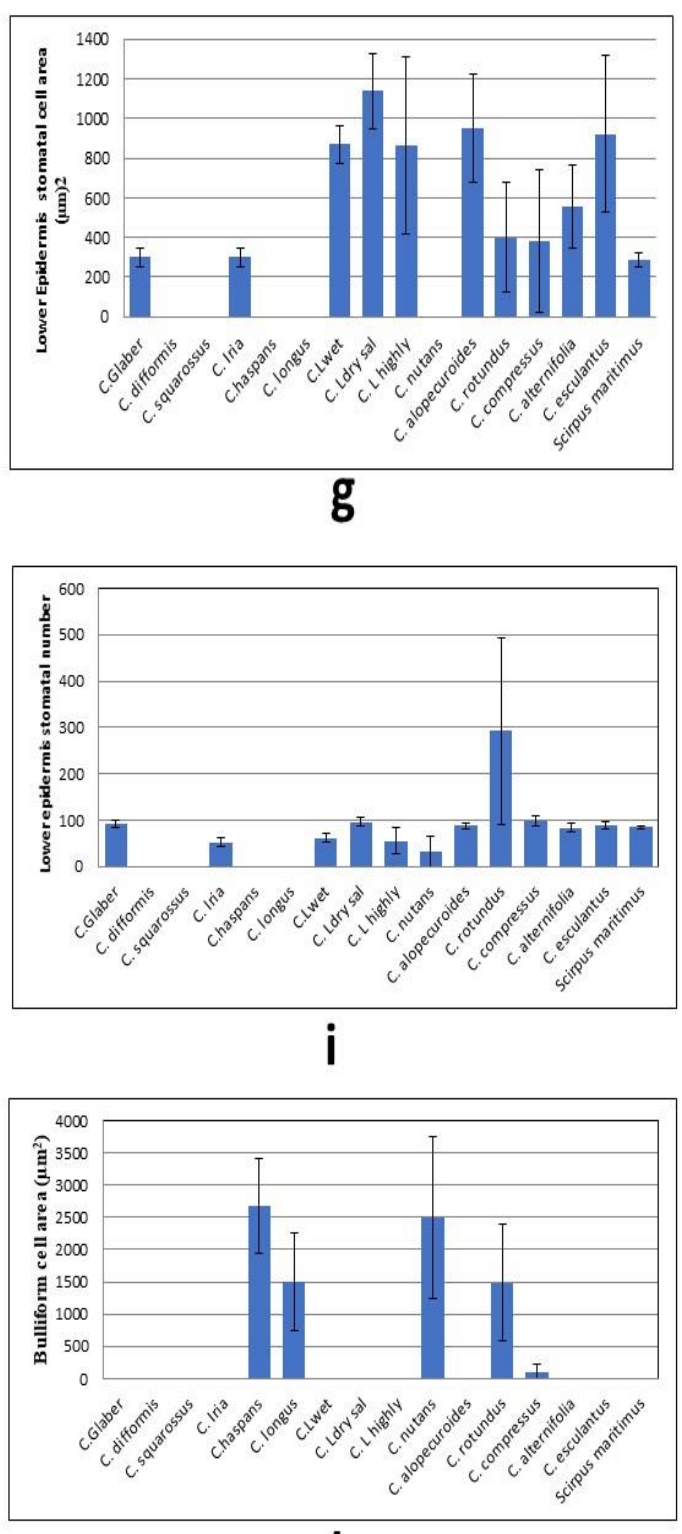

k
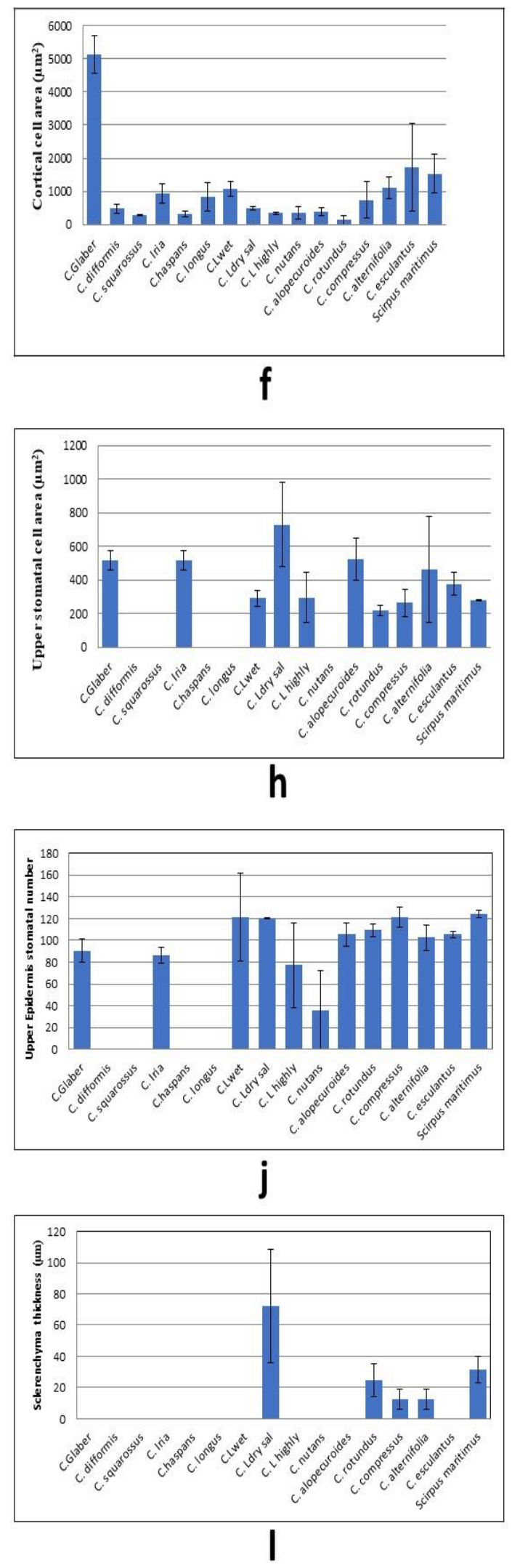


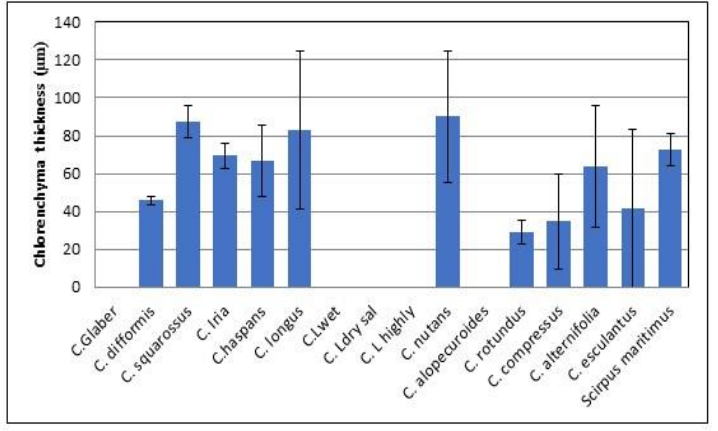

m

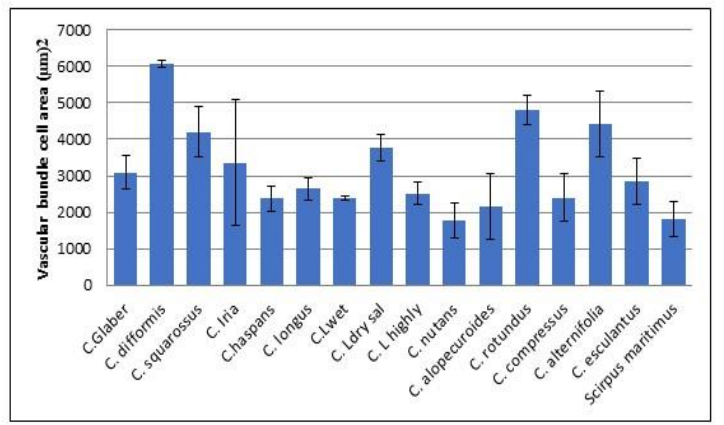

o

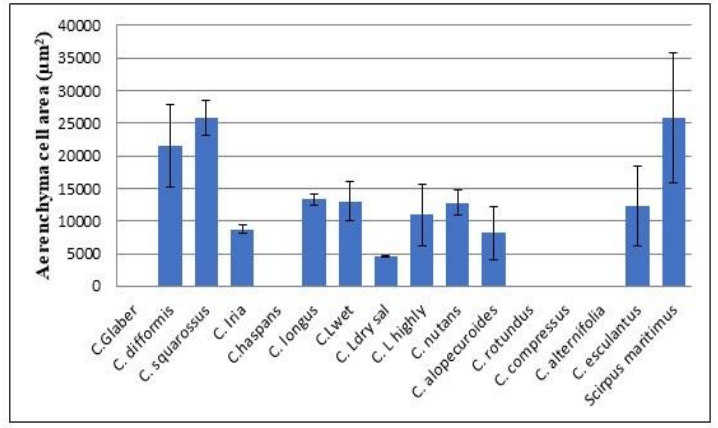

n

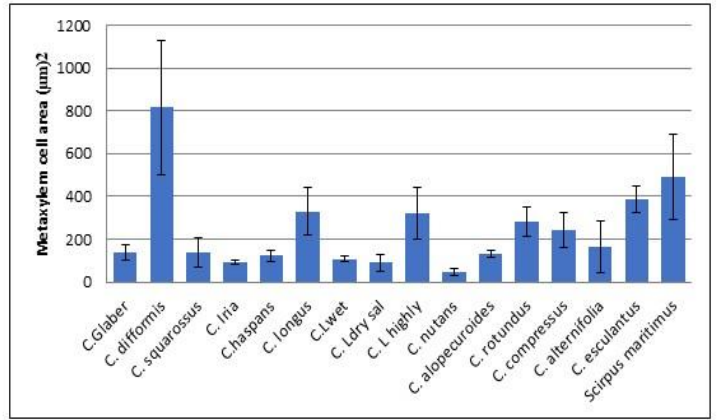

p

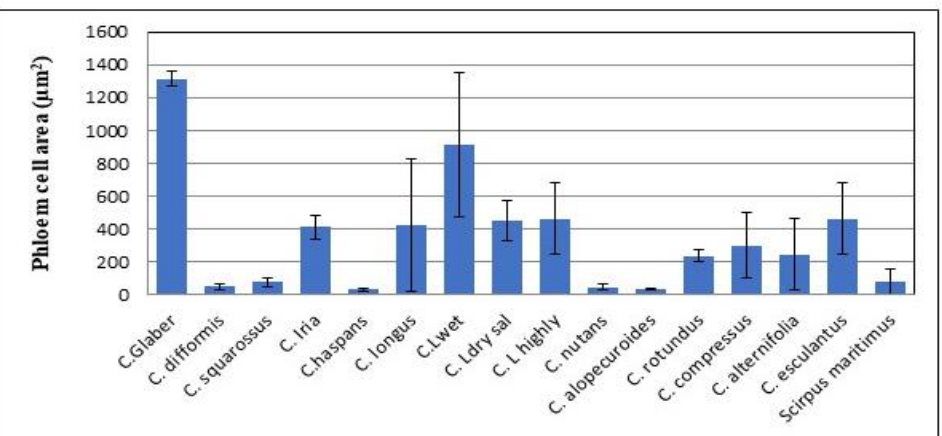

q

Figure1. Statistical Data of leaf anatomy parameters of different species of Cyperus growing in Punjab, Pakistan 
Pure Appl. Biol., 10(3):726-737, September, 2021
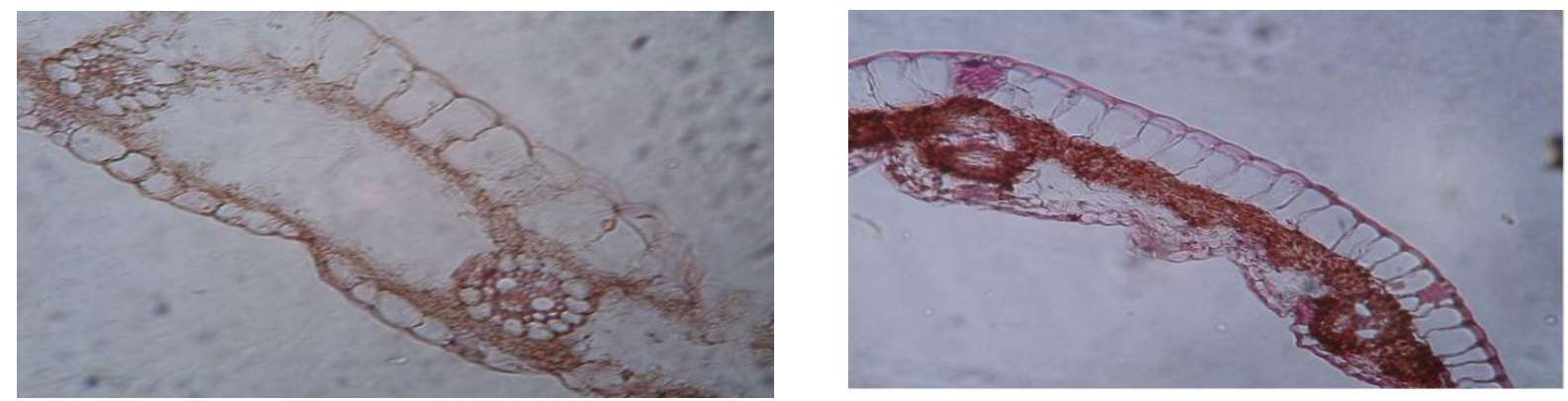

\section{Cyperusnutans}

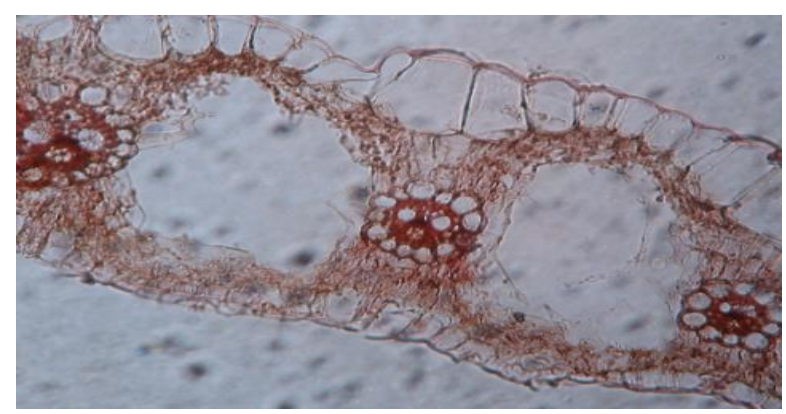

\section{Cyperussquarrosus}

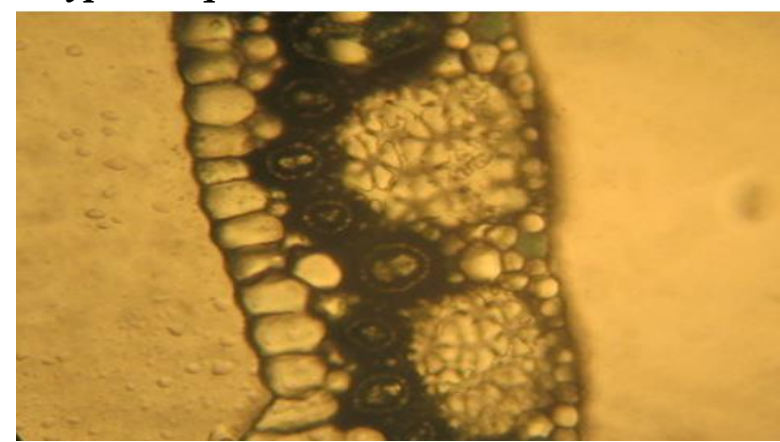

\section{Cyperusalopecuroides}

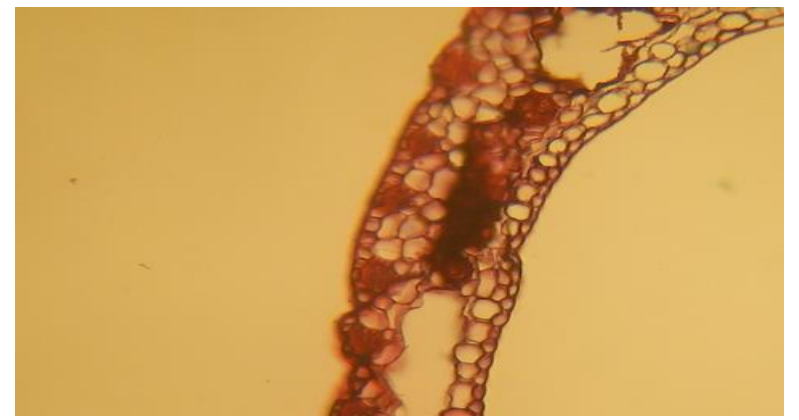

Cyperuslaevigatushighly saline

\section{Cyperusrotundus}

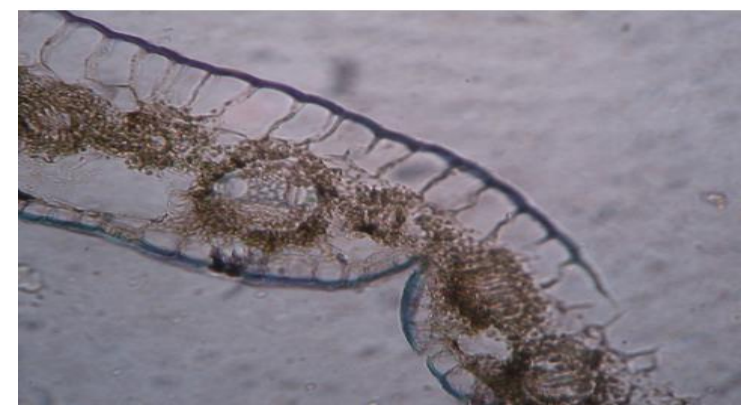

Cyperusiria

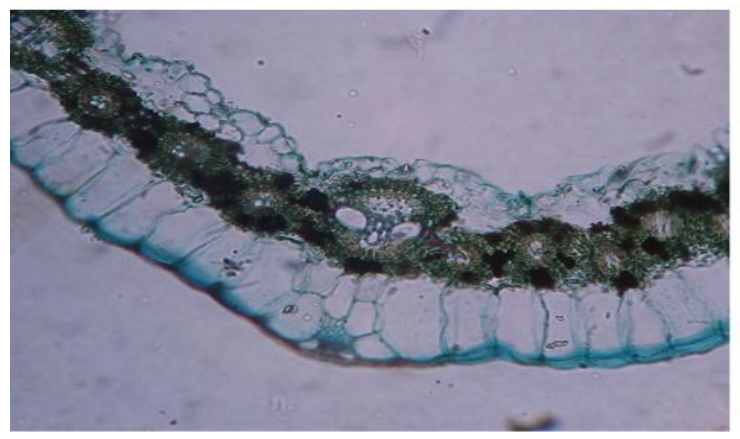

Cyperuscompressus

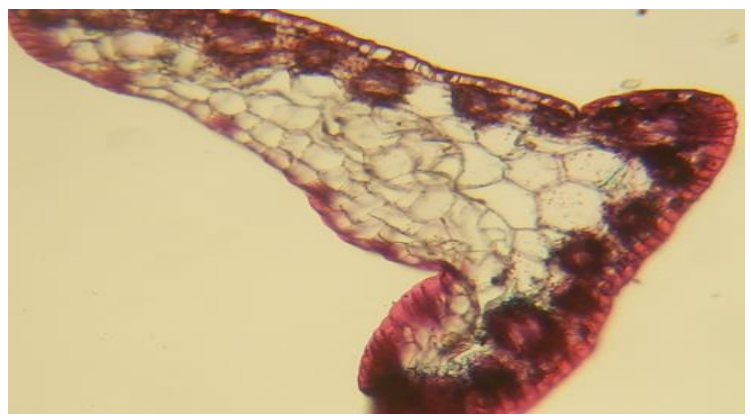

\section{Cyperusglaber}




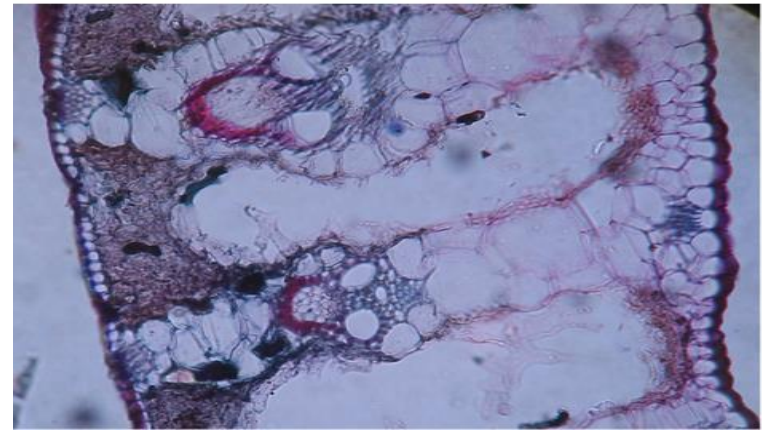

Scirpusmaritimus

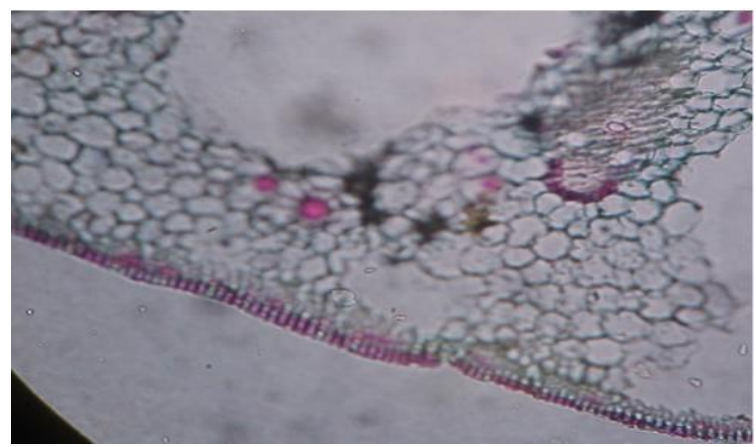

C. alternifolia

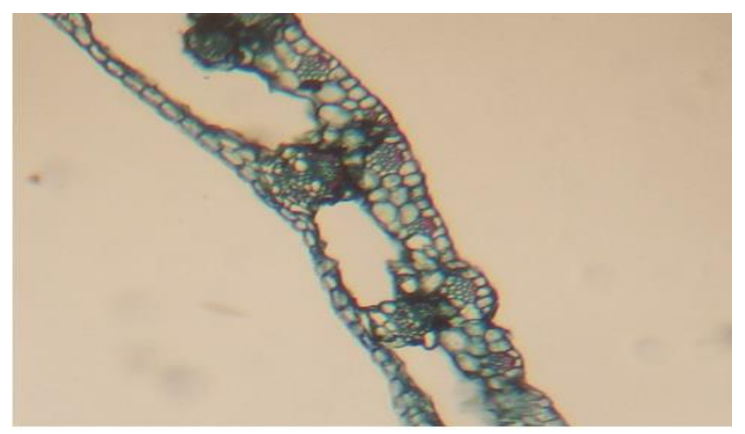

Cyperuslaevigatus Dry saline

\section{Figure 2. TS of the leaf of some species of family Cyperaceae from Punjab}

\section{Discussion}

With a diverse morphology and cosmopolitan distribution, Cyperaceae is among the core eudicots in angiosperms having a wide variety of habitats such as damp marshes, temperate forests, steep slopes, old fields and even the arctic tundra. These varieties of habitats require specific modifications in the anatomy of various parts of plants to survive the harsh climate and soil conditions of that region [10]. In this study, we focused on the leaf anatomy modifications due to different soil conditions of various regions of Punjab, Pakistan. We observed distinct leaf anatomy changes in species growing in different districts of Punjab.

Researchers have demonstrated that in areas with limited water supply, plants modify their stomata morphology to keep the evapotranspiration process continued even in short supply of water [11]. The soil of Sahianwala region of Punjab is dry and saline in nature with climate conditions similar to drought. Our study found that the $C$. laevigatus species growing in Sahianwala region have increase cell area of the stomata in the upper and lower epidermis of the leaf. This modification involving the ability to regulate the stomata opening serves to control the loss of water in the dry saline soil and hence helps in the survival of the species in that area. Other changes in leaf anatomy involve increase in sclerenchyma thickness and decrease in aerenchyma thickness to limit further loss of water. 
Table2.Leaf anatomy parameters of different species of Cyperus

\begin{tabular}{|c|c|c|c|c|c|c|c|c|c|c|c|c|c|c|c|c|}
\hline $\begin{array}{c}\text { Stem } \\
\text { Anatomical } \\
\text { Parameters }\end{array}$ & ن্ & ن & نे & ن & 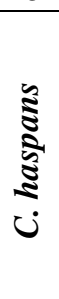 & $\frac{0}{\tilde{2}}$ & نे & 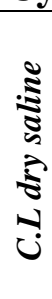 & 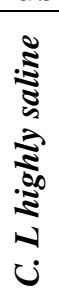 & 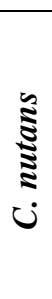 & 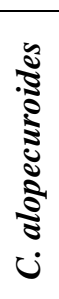 & ن & 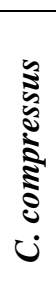 & 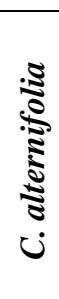 & 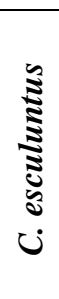 & 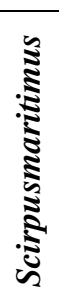 \\
\hline $\begin{array}{l}\text { Leaf area } \\
\quad(\mu \mathrm{m} 2)\end{array}$ & 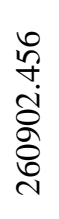 & 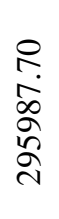 & 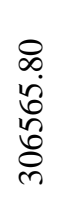 & 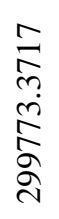 & 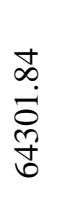 & 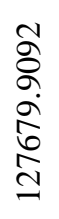 & $\begin{array}{l}\infty \\
\infty \\
\dot{0} \\
\mathbb{D}_{0} \\
\infty\end{array}$ & 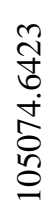 & 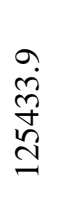 & 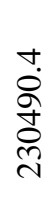 & $\begin{array}{l}\stackrel{+}{\grave{~}} \\
\stackrel{\Xi}{\sigma}\end{array}$ & $\begin{array}{l}\text { J. } \\
\text { 守 } \\
\text { वे }\end{array}$ & 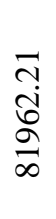 & \begin{tabular}{l}
\multirow{1}{1}{} \\
20 \\
2 \\
0
\end{tabular} & $\begin{array}{l}\hat{0} \\
\text { ठे } \\
\text { ते }\end{array}$ & $\frac{\substack{\infty \\
\infty}}{\infty}$ \\
\hline $\begin{array}{c}\text { Epidermis } \\
\text { thickness } \\
(\mu \mathrm{m})\end{array}$ & $\stackrel{m}{\stackrel{9}{a}}$ & $\begin{array}{l}\infty \\
\infty \\
\ddot{n}\end{array}$ & î̀. & 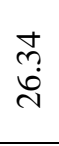 & $\stackrel{8}{\stackrel{8}{\Xi}}$ & $\underset{r}{\stackrel{r}{r}}$ & $\begin{array}{l}\hat{0} \\
0\end{array}$ & $\stackrel{0}{0}$ & $\begin{array}{l}\infty \\
\stackrel{\infty}{i} \\
\stackrel{\sim}{\sim}\end{array}$ & n? & $\frac{1}{2}$ & ָి & $\hat{\sigma}$ & $\tilde{\infty}$ & $\stackrel{+}{\circ}$ & $\stackrel{n}{n}$ \\
\hline $\begin{array}{l}\text { Epidermis } \\
\text { cell area } \\
(\mu \mathrm{m} 2)\end{array}$ & $\begin{array}{l}\vec{\nabla} \\
\stackrel{+}{\Xi}\end{array}$ & $\begin{array}{l}\infty \\
⿱ 亠 \\
\infty \\
\infty \\
0\end{array}$ & $\begin{array}{l}\text { ô } \\
\stackrel{i}{i} \\
\stackrel{\text { }}{\text { }}\end{array}$ & $\frac{\stackrel{m}{r}}{\stackrel{n}{i}}$ & 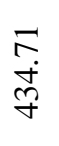 & 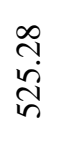 & 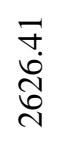 & $\begin{array}{l}\stackrel{f}{\checkmark} \\
\text { s. } \\
\text { gे }\end{array}$ & 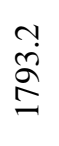 & 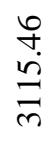 & $\begin{array}{l}\text { ¿े. } \\
\text { on } \\
\text { mे }\end{array}$ & $\frac{n}{\stackrel{n}{v}}$ & 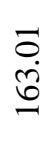 & $\stackrel{n}{\stackrel{n}{v}}$ & $\begin{array}{l}\hat{\sigma} \\
\dot{0} \\
\dot{0}\end{array}$ & $\stackrel{\substack{n \\
m}}{m}$ \\
\hline $\begin{array}{c}\text { Lower } \\
\text { Epidermis } \\
\text { cell area } \\
(\mu \mathrm{m} 2)\end{array}$ & $\begin{array}{l}\vec{\nabla} \\
\dot{\vec{J}}\end{array}$ & $\begin{array}{l}0 \\
\infty \\
\infty \\
\infty \\
\infty\end{array}$ & 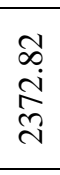 & 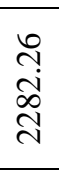 & $\underset{⿱ 亠 䒑 十}{\stackrel{F}{+}}$ & $\begin{array}{l}\stackrel{q}{+} \\
\infty \\
\text { ले }\end{array}$ & $\begin{array}{l}\infty \\
\infty \\
i \\
0\end{array}$ & $\begin{array}{l}8 \\
\frac{1}{0} \\
\infty\end{array}$ & $\begin{array}{l}\text { do } \\
\text { î } \\
\text { I }\end{array}$ & 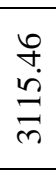 & $\underset{⿱ ㇒}{\stackrel{f}{f}}$ & $\begin{array}{l}\stackrel{8}{0} \\
\frac{\dot{0}}{\infty}\end{array}$ & $\begin{array}{l}\text { ले } \\
\text { में }\end{array}$ & $\begin{array}{l}8 \\
\stackrel{8}{0} \\
\text { in }\end{array}$ & $\begin{array}{l}0 \\
\dot{0} \\
\hat{\sigma}\end{array}$ & $\begin{array}{l}\stackrel{f}{0} \\
\stackrel{0}{\infty}\end{array}$ \\
\hline $\begin{array}{c}\text { Upper } \\
\text { Epidermis } \\
\text { Cell Area } \\
\left(\mu \mathrm{m}^{2}\right)\end{array}$ & 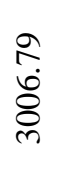 & 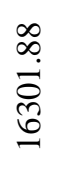 & $\begin{array}{l}\stackrel{n}{n} \\
\hat{n} \\
n\end{array}$ & \begin{tabular}{l} 
ñ \\
\multirow{8}{*}{} \\
d
\end{tabular} & 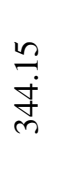 & $\begin{array}{l}\stackrel{a}{+} \\
\infty \\
\text { mे }\end{array}$ & $\begin{array}{l} \pm \\
\stackrel{\Xi}{0} \\
\text { ते }\end{array}$ & $\begin{array}{l}\text { to } \\
\text { í } \\
\text { Ṡ }\end{array}$ & $\begin{array}{l}\bar{\infty} \\
\stackrel{0}{0} \\
\text { ते }\end{array}$ & $\begin{array}{l}= \\
\dot{\infty} \\
\stackrel{2}{a}\end{array}$ & $\begin{array}{l}a \\
\hat{n} \\
\hat{n}\end{array}$ & $\begin{array}{l}\stackrel{f}{+} \\
\ddot{\infty}\end{array}$ & $\begin{array}{l}\text { ô. } \\
\text { हैं } \\
\text { nె }\end{array}$ & $\begin{array}{l}\stackrel{\alpha}{\sigma} \\
\stackrel{2}{2}\end{array}$ & $\begin{array}{l}\text { đे } \\
\text { ठ̀ }\end{array}$ & 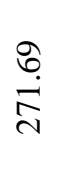 \\
\hline $\begin{array}{c}\text { Cortical Cell } \\
\text { Area }\left(\mu \mathbf{m}^{2}\right)\end{array}$ & 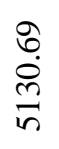 & $\begin{array}{l}8 \\
\stackrel{8}{r} \\
\frac{8}{+}\end{array}$ & $\begin{array}{l}8 \\
\dot{0} \\
\dot{2}\end{array}$ & 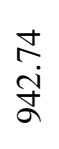 & $\begin{array}{l}\text { ले } \\
\text { ڤై } \\
\text { ले }\end{array}$ & $\begin{array}{l}\tilde{\sigma} \\
\stackrel{+}{+} \\
\infty\end{array}$ & $\begin{array}{l}= \\
\stackrel{1}{8}\end{array}$ & $\begin{array}{l}0 \\
n \\
\infty \\
o\end{array}$ & $\begin{array}{l}\hat{\alpha} \\
\text { ले }\end{array}$ & $\begin{array}{l}n \\
n \\
n \\
n\end{array}$ & 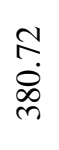 & $\begin{array}{l}\hat{a} \\
\hat{n}\end{array}$ & $\begin{array}{l}\infty \\
\infty \\
\infty \\
\infty\end{array}$ & $\begin{array}{l}\stackrel{+}{+} \\
\stackrel{0}{\Xi}\end{array}$ & $\begin{array}{c}\bar{i} \\
\stackrel{\Xi}{\Sigma}\end{array}$ & $\begin{array}{l}\text { oे } \\
\text { ลें } \\
\text { ñ }\end{array}$ \\
\hline $\begin{array}{c}\text { Lower } \\
\text { Epidermis } \\
\text { Stomatal Cell } \\
\text { Area }\left(\mu \mathbf{m}^{2}\right)\end{array}$ & $\frac{m}{2}$ & & & $\frac{m}{2}$ & & & 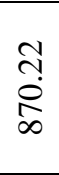 & $\begin{array}{l}\hat{n} \\
\stackrel{n}{2} \\
=\end{array}$ & $\begin{array}{l}0 \\
\dot{0} \\
\infty\end{array}$ & & $\stackrel{\infty}{n}$ & $\begin{array}{l}n \\
\infty \\
\infty \\
\infty\end{array}$ & $\begin{array}{l}\text { I. } \\
\stackrel{0}{\infty} \\
\infty\end{array}$ & $\begin{array}{l}a \\
i \\
n \\
n\end{array}$ & $\begin{array}{l}\infty \\
\stackrel{0}{0} \\
\text { ๙ }\end{array}$ & $\begin{array}{l}\Delta \\
n \\
i \\
\infty \\
i\end{array}$ \\
\hline
\end{tabular}


Mumtaz et al.

\begin{tabular}{|c|c|c|c|c|c|c|c|c|c|c|c|c|c|c|c|c|}
\hline $\begin{array}{c}\text { Upper } \\
\text { Stomatal Cell } \\
\text { Area }\left(\mu \mathbf{m}^{2}\right)\end{array}$ & $\begin{array}{l}\stackrel{a}{0} \\
\stackrel{0}{n}\end{array}$ & & & $\begin{array}{l}\stackrel{a}{6} \\
\text { in }\end{array}$ & & & $\begin{array}{l}\text { के } \\
\text { ¿ें }\end{array}$ & 홈 & $\begin{array}{l}0 \\
\stackrel{+}{\mathbf{N}}\end{array}$ & & 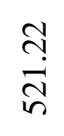 & $\begin{array}{l}\stackrel{n}{?} \\
\stackrel{n}{a}\end{array}$ & 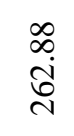 & $\begin{array}{l}\text { mij } \\
\text { b }\end{array}$ & 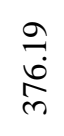 & $\begin{array}{l}\stackrel{7}{a} \\
\stackrel{\Delta}{\infty}\end{array}$ \\
\hline $\begin{array}{c}\text { Lower } \\
\text { Epidermis } \\
\text { Stomatal } \\
\text { Number } \\
\end{array}$ & $\stackrel{m}{n}$ & & & in & & & ธ్ర & $\begin{array}{l}: \\
\stackrel{8}{0} \\
\stackrel{0}{\circ}\end{array}$ & $\begin{array}{l}: \\
: \\
i n\end{array}$ & $\stackrel{m}{m}$ & $\begin{array}{c}m \\
\infty \\
\infty \\
\infty\end{array}$ & $\begin{array}{l}\hat{a} \\
\text { à } \\
\text { in }\end{array}$ & $\stackrel{\infty}{\circ}$ & $\Phi$ & $\begin{array}{l}: \\
\substack{\infty \\
\infty \\
\infty}\end{array}$ & $\mathscr{\infty}$ \\
\hline $\begin{array}{c}\text { Upper } \\
\text { Epidermis } \\
\text { Stomatal } \\
\text { Number } \\
\end{array}$ & $\begin{array}{l}\stackrel{8}{0} \\
\stackrel{8}{\circ}\end{array}$ & & & 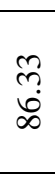 & & & 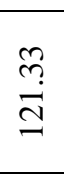 & $\begin{array}{l}\stackrel{0}{0} \\
\stackrel{\Xi}{\simeq}\end{array}$ & 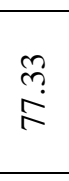 & ల్ల & 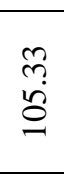 & $\begin{array}{c}\stackrel{m}{2} \\
\stackrel{g}{a}\end{array}$ & 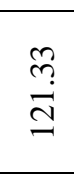 & $\begin{array}{l}\stackrel{0}{0} \\
\stackrel{\delta}{0}\end{array}$ & 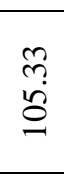 & $\stackrel{\Xi}{I}$ \\
\hline $\begin{array}{l}\text { Bulliform cell } \\
\text { area }(\mu \mathrm{m} 2)\end{array}$ & & & & & 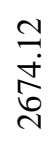 & $\begin{array}{l}\stackrel{a}{0} \\
\dot{a} \\
\text { वे }\end{array}$ & & & & 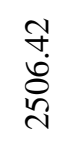 & & 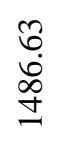 & $\begin{array}{l}\text { F } \\
\stackrel{\infty}{0} \\
\stackrel{0}{0}\end{array}$ & & & \\
\hline $\begin{array}{c}\text { Sclerenchym } \\
\text { a Thickness } \\
(\mu \mathrm{m})\end{array}$ & & & & & & & & $\vec{i}$ & & & & $\begin{array}{l}\stackrel{+}{+} \\
\stackrel{+}{+}\end{array}$ & 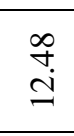 & $\stackrel{\infty}{\stackrel{\infty}{\leq}}$ & & $\frac{\pi}{m}$ \\
\hline $\begin{array}{c}\text { Collenchyma } \\
\text { Thickness } \\
(\mu \mathrm{m})\end{array}$ & & $\begin{array}{l}\stackrel{0}{i} \\
i g\end{array}$ & $\begin{array}{c}0 \\
\stackrel{\infty}{?} \\
\infty\end{array}$ & ले. & 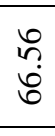 & $\overbrace{\infty}^{\infty}$ & & & & $\stackrel{m}{\circ}$ & & $\frac{i}{3}$ & $\begin{array}{l}8 \\
\dot{0} \\
\dot{m}\end{array}$ & $\stackrel{\infty}{\stackrel{\infty}{\sigma}}$ & $\begin{array}{l}\stackrel{0}{\dot{\sigma}} \\
\end{array}$ & $\stackrel{\infty}{i}$ \\
\hline $\begin{array}{l}\text { Aerenchyma } \\
\text { Cell Area } \\
\left(\mu \mathbf{m}^{2}\right)\end{array}$ & & $\begin{array}{l}5 \\
\infty \\
\infty \\
\stackrel{\sim}{+}\end{array}$ & 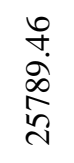 & 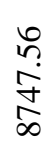 & & $\begin{array}{l}\text { ì } \\
\text { तิ } \\
\text { dు }\end{array}$ & $\begin{array}{l}\text { D. } \\
\text { o. } \\
\text { d. }\end{array}$ & 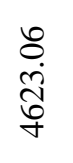 & $\begin{array}{l}a \\
\vdots \\
\dot{a} \\
0 \\
0\end{array}$ & $\begin{array}{l}\text { ते } \\
\text { ¿े } \\
\text { త్ }\end{array}$ & $\begin{array}{l}n \\
m \\
\infty \\
\infty \\
\infty\end{array}$ & & & & 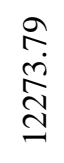 & $\begin{array}{l}\stackrel{2}{+} \\
\stackrel{d}{\infty} \\
\infty \\
\end{array}$ \\
\hline $\begin{array}{l}\text { Vascular } \\
\text { Bundle Cell } \\
\text { Area }\left(\mu \mathrm{m}^{2}\right)\end{array}$ & 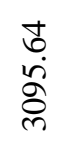 & 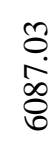 & $\begin{array}{l}\stackrel{\infty}{+} \\
\stackrel{\sigma}{i} \\
\stackrel{\nabla}{\sigma}\end{array}$ & बे & 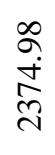 & $\begin{array}{l}\stackrel{+}{i} \\
\text { id } \\
\text { di }\end{array}$ & 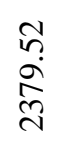 & 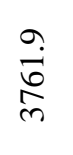 & 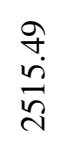 & 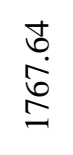 & 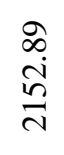 & 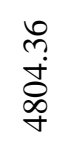 & $\underset{\substack{i \\
\stackrel{o}{d}}}{\infty}$ & $\vec{a}$ & 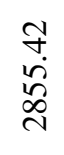 & $\begin{array}{l}\stackrel{?}{+} \\
\stackrel{0}{0} \\
\stackrel{\infty}{\sim}\end{array}$ \\
\hline $\begin{array}{c}\text { Metaxylem } \\
\text { Cell Area } \\
\left(\mu \mathbf{m}^{2}\right)\end{array}$ & $\begin{array}{l}\hat{a} \\
\text { ch } \\
\end{array}$ & $\begin{array}{l}\tilde{\infty} \\
\stackrel{\omega}{\infty} \\
\infty\end{array}$ & $\begin{array}{l}\hat{a} \\
\hat{b} \\
\end{array}$ & $\begin{array}{l}\text { ț } \\
\stackrel{8}{\circ}\end{array}$ & $\begin{array}{l}\hat{0} \\
\text { ì } \\
\text { }\end{array}$ & 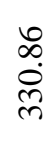 & $\begin{array}{l}\stackrel{R}{A} \\
\stackrel{\infty}{g}\end{array}$ & $\begin{array}{l}\text { ț } \\
\stackrel{8}{\circ}\end{array}$ & $\stackrel{\infty}{\vec{j}}$ & $\begin{array}{l}\mathscr{b} \\
\ddot{q} \\
\ddot{g}\end{array}$ & $\frac{f}{\dot{m}}$ & $\underset{\vec{D}}{\overrightarrow{\mathrm{d}}}$ & \begin{tabular}{l}
$\stackrel{n}{f}$ \\
\multirow{j}{d}{} \\
$d$
\end{tabular} & 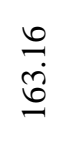 & 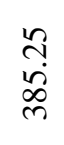 & 吕 \\
\hline $\begin{array}{l}\text { Phleom Cell } \\
\text { Area }\left(\mu \mathrm{m}^{2}\right)\end{array}$ & 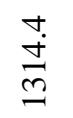 & $\begin{array}{l}\infty \\
\infty \\
\dot{q}\end{array}$ & $\stackrel{\substack{0 \\
i}}{i}$ & $\begin{array}{l}\text { If } \\
\text { di }\end{array}$ & $\stackrel{\mathbb{m}}{m}$ & $\frac{\vec{n}}{\vec{\gamma}}$ & 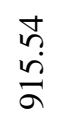 & $\begin{array}{l}\stackrel{+}{N} \\
\stackrel{\sim}{y}\end{array}$ & $\begin{array}{l}\text { î̀ } \\
\text { ơ }\end{array}$ & $\begin{array}{l}\stackrel{d}{0} \\
\dot{y}\end{array}$ & $\begin{array}{l}\text { त̂ } \\
\text { d. } \\
\text { n. }\end{array}$ & 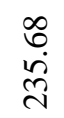 & $\frac{m}{\vec{a}}$ & 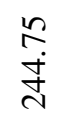 & 垈 & $\stackrel{\infty}{n}$ \\
\hline
\end{tabular}


Modifications in the cortex are also associated with harsh soil conditions. Cortex is involved in the storage of carbohydrates and ultimately in maintaining the water-pull by the transpiration stream. C. glaber species in the Head Rasool region of Punjab also show leaf anatomy modifications of larger cortical cell area and larger phloem cell area along with thick epidermis which indicate the modifications in response to harsh soil conditions of the area. Another species $C$. squarossus shows increase in the aerenchyma cell area which shows that there is a diversity of species in the Head Rasool region. This diversity can be attributed to difference in the soil conditions suitable for different types of Cyperus species.

Studies have shown that highly saline soil conditions can also affect the number of leaves, leaf area, and plant fresh weight. The growth of the leaf area is an important factor in determining the plant's transpiration, light interception, and overall productivity. Other modifications observed in the leaf anatomy of the species include increased thickness of epidermis and reduction of upper epidermal cell area, increase in the number of stomata on the upper epidermis, larger aerenchyma cell area and reduced stomatal cell area on lower epidermis. All these modifications can help the plant in preventing excessive water loss during transpiration and absorbing more light to undergo rapid photosynthesis for keeping the plant growth unaffected despite the high saline soil conditions [12].

C. compressus found in the highly saline region of KalarKahar also shows anatomical modifications in the leaf in the form of the thinnest epidermis and smallest bulliform cell area. While on the other hand, C. haspens in the Chiniot district has the largest bulliform cell area with minimum phloem cell area in its leaves.

The city of Faisalabad has dry loamy soil with little rain and hence can be thought of as a waterdeficient area for plants. Probably, to meet this water shortage, $C$. alternifolia collected from the botanical garden of the University of Agriculture, Faisalabad, has the smallest leaf area among all the species in Punjab. The small leaf area can prevent the loss of water from transpiration. The species also show minimum sclerenchyma thickness in leaves.
Similarly, another species sample C. rotundus collected from the same university demonstrated large vascular bundle cell area, minimum cortical cell area, thin collenchyma, minimum upper epidermal stomatal cell area, and increased number of stomata on lower epidermis.

Cyperus species collected from the Jarhanwala region showed anatomical changes in the form of maximum upper epidermal cell area, maximum metaxylem cell area and maximum vascular bundle cell area.

Another region explored in Punjab is Khari Mural where $C$. nutans which showed leaf modifications in the form of thickest collenchyma, large lower epidermal cell area, a minimum number of stomata on the upper and lower epidermis, thick bulliform cell area, small vascular bundle cell area, and minimum metaxylem cell area.

Likewise, C. esculuntus samples collected from the Pakkana region showed leaf anatomy with minimum epidermal cell area and minimum lower epidermal cell area. The maximum epidermal cell area was shown by $C$. alopecuroides collected from the Balloki region of Punjab.

After analyzing all the soil samples and comparing the leaf anatomies of different species, we could relate that the modifications in different cells of the leaves in Cyperaceae are the cause behind the survival and growth of its species in such a variety of soil habitats in Punjab. This is a small-sized study focusing only on the analysis of the transverse sections of selected species in Punjab. However, further elaborated studies can be planned for the detailed analysis and comparison of leaf modifications in Cyperus plants with respect to different mineral ion concentrations, salinity, and moisture in the soil [13].

\section{Conclusion}

Soil structure and composition can affect the survival and growth of a plant. For this purpose, the plant undergoes specific modifications to cope with the challenges of the harsh climate and soil conditions. The Cyperaceae species distributed in various soil regions of Punjab also undergo different leaf anatomy modifications such as changes in leaf area, different thickness of the upper and lower epidermis, changes in the number of stomata on the upper and lower epidermis, 
variations in the size of vascular bundle cell area, bulliform cell area, metaxylem, and phloem cell area and finally altered thickness of sclerenchyma, collenchyma, and aerenchyma in leaves in order to meet the water and light requirements of the plant. Cyperaceae species with a greater number of modifications show a higher growth rate even in unfavorable soil and climate conditions while plants with moderate or no modifications show limited growth in those areas. This study showed dispersal of selected species of Cyperus genus in different soil regions of Punjab with respect to their leaf anatomy modifications.

\section{Authors' contributions}

Conceived and designed the experiments: A Teseen \& A Ameer, Performed the experiments: A Taseen \& A Ameer, Analyzed the data: A Mumtaz, Contributed materials/ analysis/ tools: A Mumtaz, Wrote the paper: A Mumtaz.

\section{Acknowledgment}

The authors of this study acknowledge and appreciate the efforts of all participants involved.

\section{References}

1. Besnard G, Muasya AM, Russier F, Roalson EH, Salamin N \& Christin PA (2009). Phylogenomics of $\mathrm{C} 4$ Photosynthesis in Sedges (Cyperaceae): Multiple Appearances and Genetic Convergence. Mol Biol Evol 26(8): 1909-1919.

2. Blaser HW (1941). Studies in the Morphology of the Cyperaceae. I. Morphology of Flowers. A. Scirpoid Genera. Am J Bot 28(7): 542-551.

3. Besnard G, Muasya AM, Russier F, Roalson EH, Salamin N \& Christin PA (2009). Phylogenomics of C4 Photosynthesis in Sedges (Cyperaceae): Multiple Appearances and Genetic Convergence. Mol Biol Evol 26(8): 1909-1919.

4. Escudero M, Hipp AL, Waterway MJ \& Valente LM (2012). Diversification rates and chromosome evolution in the most diverse angiosperm genus of the temperate zone (Carex, Cyperaceae). Mol Phylogenet Evol 63(3): 650-655.
5. Carolin RC, Jacobs SWL \& Vesk M (1977). The Ultrastructure of Kranz Cells in the Family Cyperaceae. Bot Gaz 138(4): 413419.

6. Butt MA, Zafar M, Ahmad M, Sultana S, Ullah F, Jan G \& Naqvi S (2018). Morphopalynological study of Cyperaceae from wetlands of Azad Jammu and Kashmir using SEM and LM.Microsc Res Techniq 81(5): 458-468.

7. Ikram S, Bhatti KH \&ParvaizM (2014). Ethnobotanical studies of aquatic plants of district Sialkot, Punjab (Pakistan). J Med Plants 2(1): 58-63.

8. Condom N, Kuper M, Marlet S, Valles V \&Kijne J (1999). Salinization, alkalinization and sodification in Punjab (Pakistan): characterization of the geochemical and physical processes of degradation. Land Degrad Dev 10(2): 123-140.

9. Hassan A, Ijaz SS, Lal R, Ali S, Hussain Q, Ansar M \&Baloch MS (2016). Depth distribution of soil organic carbon fractions in relation to tillage and cropping sequences in some dry lands of Punjab, Pakistan. Land Degrad Dev 27(4): 1175-1185.

10. Metcalfe CR (1969). Anatomy as an aid to classifying the cyperaceae. Am J Bot 56(7): 782-790.

11. Ueno O \& TakedaT (1992). Photosynthesis pathways, ecological characteristics, and the geographical distribution of the Cyperaceae in Japan. Oecologia 89(2): 195-203.

12. Xu Z\& Zhou G (2008). Responses of leaf stomatal density to water status and its relationship with photosynthesis in a grass. $J$ Exp Bot 59(12): 3317-3325.

13. Koester RP, Skoneczka JA, Cary TR, Diers BW \& Ainsworth EA (2014). Historical gains in soybean (Glycine max Merr.) seed yield are driven by linear increases in light interception, energy conversion, and partitioning efficiencies. J Exp Bot 65(12): 3311-3321. 